\title{
The Necessity and Possibility of the Effective Abutting Joint between Linguistics and Chinese Teaching
}

\author{
Xiao Jiugen ${ }^{1, a}$, ${ }^{2}$ Wenjuan Zhang ${ }^{2, b}$ Chen Xijuan ${ }^{3, c}$ \\ 1,2 Jiangxi Normal University, School of Literature, Language and Language Life Research Center, \\ Nanchang, Jiangxi, China \\ ${ }^{3}$ Nanchang Institution of Technology and Science, Nanchang, Jiangxi, China \\ a jxsdxjg666666@sina.com, b1445527062@qq.com ,c403744677@qq.com \\ * Xiao Jiugen
}

Key words: linguistics, Chinese teaching, abutting joint, necessity, possibility

\begin{abstract}
Chinese teaching is very close, so linguistics can be introduced into the practice of the basic Chinese teaching. The effective abutting joint is not only with its necessity and possibility, but also urgency. As it is, the joint will promote the development of Chinese education in China. However, according to the survey, there is still a serious gap between the two. Considering all kinds of favorable factors and the function of linguistics in Chinese teaching, it is not difficult to find that, in the practice of Chinese teaching, it is entirely possible to achieve effective abutting joint with sufficient conditions.
\end{abstract}

\section{语言学与语文教学实现有效对接的必要性和可能性}

\author{
肖九根 $1, \mathrm{a}$ 张文娟 $2, \mathrm{~b}$ 陈曦娟 $3, \mathrm{c}$ \\ (1. 2. 江西师范大学文学院, 语言与语言生活研究中心, 江西南昌, 中国; ${ }^{3}$. 南昌工学院, 江西南昌, \\ 中国）
}

Emai1: ajxsdxjg666666@sina. com(肖九根),b1445527062@qq. com（张文娟）,c 403744677@qq. com(陈 曦娟）

关键词: 语言学; 语文教学; 实现对接; 必要性; 可能性

摘 要: 语言学与语文教学关系十分密切, 将其引入基础语文教学实践中, 以实现有效对接, 不仅有其必要性和可能性, 而且还显得十分迫切。诚如是, 必将推动我国语文教育教学的大 发展。然而, 据调查所知, 目前语文教学的现状依然存在着二者严重脱节的现象。如果综观 各种有利因素以及语言学在语文教学中所起的作用, 就不难发现: 在语文教学实践中, 实现 二者有效对接已经具备充足条件，完全有可能的了。

\section{1. 引言}

语言文字是人类最重要的交际工具和信息载体，是人类文化的重要组成部分， ${ }^{[1]}$ 其指向 是人的一种基本生存能力, 也是人作为人的一种基本标志。一个人小学之前就已开始了这方 面的学习, 当完成中学阶段学习之后, 无论从事什么职业, 他所具有的这种能力就可以发挥作 用, 而且这种作用还将延续下去, 以至终身。如果没有这方面的能力, 又不继续学习, 任何人 都无法在这个社会正常地生存, 更不可能为社会尽其能而展其才, 所以我们的语文教学应致 力于培养学生的语言文字运用能力。但是, 审视当下的语文教学, 我们不难发现, 在这个信 息高度发达、民族全力创新的时代, 语文教学还是依旧沿着一条去语言理论知识的道路走着, 
最终导致了语文教学与语言基础理论知识空前疏离的严重现象。上世纪末叶之初, 语文教学 界质疑语言学理论在语文教学中的作用, 从此语言理论、语言知识渐渐地从语文教学领域里 淡出。正是基于这一点, 我们根据实地调查的基本数据, 从语言学与语文教学二者存在严重 脱节的实际情况出发, 探讨语言学与语文教学实现有效对接的必要性和可能性, 以期为之提 供一定的理论依据。

\section{2. 语言学与语文教学实现有效对接的必要性}

从语言学在语文教学中的缺位现状及其在语文教学中所起的作用来看, 语言学与语文教 学实现有效对接有其迫切性和必要性。实践证明, 语言理论知识已经渗透到语文教学内容的 每个方面, 而语文教学实践也离不开语言学理论的指导作用。

\section{1. 语言学在语文教学中缺位现状显示其迫切性}

语言学是当今社会科学中一门领先学科, 其研究方法以及研究成果往往被其他社会科学 的学科所借鉴。与其他社会科学的学科相比, 基础语文教学与语言学这一科学的联系更为紧 密, 二者都与语言运用有着密切的关系, 其有所不同的是语言学重在理论建设, 而语文教学 则重在实际应用。正因为这样, 语文教学应从语言学中汲取营养, 如有的学者所指出的那样: “语言教学需要建立在对语言的理性认识上, 是语言成果最直接的应用。” ${ }^{[2]}$ 但目前的实际 情况并非这样, 不少教师对语言学还缺乏理性认识, 从而导致了语文教师在其教学中忽略了 这方面的内容。例如，我们向教师问卷调查中有 1 项是:

\section{语文教学实践中, 您是否自觉地运用语言学理论知识来解决实际问题?}
(1) 经常
(2) 有时
（3）很少
(4) 从未

调查结果显示：46 位教师中有 31 位选择 “有时”，8 位选择 “经常”，6 位选择 “很少”, 1 位选择 “从未”。而实际情况与这还有出入, 不容乐观。

在语文教学过程中, 学生获取知识尤其是语言理论知识, 多是教师脱离语文教学实际而 孤立地讲授。如调查所示, 教师很少自觉地将语言理论知识应用于语文教学实践之中, 这就 使得学生大多不会熟练运用语言理论知识来解决实际问题。同样, 我们对学生问卷调查中也 有这么 1 项:

你会熟练地运用语言理论知识解决实际问题吗?
(1) 熟练
(2) 不熟练
（3）根本不会

接受调查的 264 位学生中, 66.7\%不会正确运用语言理论知识去解决实际问题。本来语文 是语言文字之学, 它 “起到规范语言, 纯化语言, 促进语言发展的作用”, [3]它的特殊任务 就是培养学生正确理解和运用语言文字的能力。而据教师讲, 仅部分学生语言理论知识扎实, 语言规范得体, 表达清晰流畅; 多数基础薄弱, 其作文普遍存在错别字、词不达意、文句不 通、标点乱用等现象。由此可见, 掌握语言理论知识不仅是培养学生言语能力的手段和中介, 还是语文教学中一项最重要、最基本的内容, 这是保证语文教学质量的根本。因此, 在语文 教学过程中, 加强语言理论知识的教学与应用, 实现语言学与语文教学对接, 不仅很有必要, 而且也是十分重要的。

\section{2. 语言学在语文教学中所起的作用显示其必要性}

张志公先生指出: “语言理论的知识系统是从小到大的: 语素一词一词组一句子一句组 一篇章; 而实际运用语言却恰恰相反, 是从大到小再回到整体的。” [3]从中不难看出, 尽管 二者顺序截然相反, 但是语素、词、词组、句子、句组、篇章等语言或言语单位是语言学与 语文教学二者共同研究的对象。因此, 语文教学完全可以而且非常有必要借助于语言学的理 论知识及其研究成果为我所用, 以加快语文教学科学化的进程。

那么, 语言学在语文教学中的作用有哪些呢? 主要体现在以下几方面: 


\section{2. 1. 在语音方面所起的作用}

语文教学主要目标之一, 就是要使学生具有正确运用语言表达的基本能力, 其中既指书 面写作，也指口语表达。口语表达则与语音息息相关，而语音学研究的成果对于学生掌握、 运用民族共同语具有十分重要的意义。首先, 语音学对音素发音特征的分析有助于方言区学 生掌握共同语的标准音。例如, 南方学生 $[\mathrm{n}]$ 、 [ I ] 普遍不分, 两个音要么读 $[\mathrm{n}]$, 要么读 $[\mathrm{I}]$, 听力上也分辨不清。要掌握好这两个音, 首先必须了解其发音的区别特征, 如果缺乏语音学 理论知识, 就难以解决这个问题。语音学表明, [n] 为舌尖中鼻音, 发音时舌尖抵住上齿龈, 形成阻碍, 气流从鼻腔冲出; 而 $[\mathrm{I}]$ 是边音, 发音时舌尖抵住上齿龈, 气流从舌头两边发出。 只有弄清楚了二者的发音部位、发音方法, 才能掌握其发音特征, 而这又是语言学中一个重 要的理论知识内容。可以这样说, 语言学的理论知识实际上渗透到语文教学中的方方面面。

2. 2. 2. 在语法方面所起的作用

语法是语言的组织规则, 它对语言理解和表达有着举足轻重的作用。语法研究可以使我 们了解语言的结构规律，了解句法结构与意义联接规律，进而更好地解决实际问题。

检阅语文高考试卷, 我们发现学生错误率最高的是语病。如 2016 年有道真题是这样的:

下列各向中，没有语病的一向是（）

A. 近日刚刚建成的西红门创业大街和青年创新创业大赛同步启动，绿色设计和 “互 联网+农业”设计是本次赛事的两大主题。

B. 最近几年，从中央到地方各级政府出台了一系列新能源汽车扶持政策，节能环

保、经济实惠的新能源汽车逐渐进入老百姓的生活。

C. 实时性是以互联网为载体的新媒体的重要特点, 是通过图片、声音、文字对新 近发生和正在发生的事件进行传播的。

D. 广西传统文化既具有典型的本土特色，又兼有受中原文化、客家文化、湘楚文 化共同影响下形成的其他特点。

通过分析我们知道，A 项 “创业大街同步启动” 主谓不宜搭配; C 项暗换主语，前半句 的主语是 “实时性” ，后半句换成了主语 “新媒体” ; D 项 “受中原文化、客家文化、湘楚 文化共同影响下” 几种句式杂糅在一起, 去掉 “受” 或 “下”。所以, 选项 B 是正确的。这 道题涉及的句法错误, 实际上都是语言学理论知识中的基本问题一一搭配不当, 成分残缺, 句式杂糅。

所以, 语文教学中, 如果教师运用语言学理论知识, 并将方法授之于学生, 训练他们的 分析能力, 毫无疑问, 许多实际问题都能迎刃而解。

2. 2. 3. 在语用方面所起的作用

语用所研究的是一定语境中语言使用时体现出来的具体意义，其核心有二：一是意义， 二是语境。 ${ }^{[4]}$ 也就是说, 它研究语言理解和使用, 研究特定情景中的特定话语, 研究如何通 过语境来理解和使用语言。由于其涉及的内容广泛, 我们仅以语用理论中的会话原则为例进 行说明。“言外之意” 是文学作品中普遍存在的现象, 语文教学时要解释清楚它的产生机制, 就必须借助于会话原则。

例如，高中语文《红楼梦》“林黛玉进贾府”里，写到贾母问黛玉念过什么书，黛玉回 答说: “只刚念了 ‘四书”。”而黛玉问姊妹们读何书时, 贾母却说: “读的是什么书, 不 过是认得两个字，不是争眼的瞎子罢了。” 此时，黛玉意识到自己实话实说已有不谦之嫌了。 所以, 当后来宝玉问黛玉可曾读书时, 她便说: “不曾读, 只上了一年学, 些须认得几个字。” 如果按美国语言学家格赖斯（Grice）的 “合作原则”，黛玉对宝玉的回答显然是不真实的, 这无疑违背了 “合作原则” 中的 “质准则”。 ${ }^{[5]}$ 但是, 读者透过黛玉刻意贬低自己的话语, 却能窥见她刚来等级森严的外祖母家时 “步步留心, 时时在意” 的心理; 与此同时, 从其言 谈举止中, 也能充分表明黛玉的教养非同一般, 其敏感、自尊的性格无形中得到了强化, 读 
者由此可以获得一种言语表达艺术美的享受。

含蓄历来是汉民族崇尚的表达方式, 日常生活中人们为了达到某一目的而有意违背语用 原则, 这样的事例屡见不鲜。听话者若要正确把握说话者的 “言外之意”，除了掌握语音、 词汇、语法等语言知识之外, 往往还需根据社会文化背景、特定语言环境等因素才能领悟其 话语真实而准确的含义。

上述例子说明, 语文教学本质上就是语用教学。 ${ }^{[6]}$ 也就是说, “语用教学就是师生在使 用语言进行交际的动态过程中发展语言和自身的语文教学。” ${ }^{[7]}$ 而语用是语言学中一个重要 的理论分支, 因此语文教学归根结底离不开语言学理论的指导。

\section{3. 语言学理论与语文教学实现有效对接的可能性}

语言学与语文教学实现有效对接既有新兴学科发展的实践经验, 又有国家教育政策的大 力扶持, 此外还有一个重要条件就是人才资源的可靠保证。因此, 在语文教学实践中, 将语 言学与之对接起来完全是有可能的。

\section{1. 语言新兴学科的快速发展为其对接提供了实践经验}

毫无疑问, “语言学是研究语言的科学”, ${ }^{[2]}$ 也就是要研究语言的基本原理, 诸如语言的 结构规律和演变规律。尽管如此, 但其一项重要的任务就是要阐述语言最首要的、也是最基 本的功能一一工具性、应用性。因此, 以语言作为研究对象的语言学也相应地分为两大部类: 理论语言学与应用语言学。理论语言学主要研究语言本体现象, 其目的是让人们了解、掌握 有关语言的理论知识; 而应用语言学则运用语言学及其相关学科的理论、方法和研究成果阐 明和解决其他领域中的语言问题, 其目的是把语言学的基础研究成果转化为社会效益, 它不 仅关注语言动态的发展, 而更为重要的是注重言语的实际应用。近些年来, 语言学家们在言 语和言语行为的广衰土地上, 开拓进取, 建立起一批批以言语研究为中心的新兴学科, 如语 用学、篇章语言学、语体学、功能主义语言学、交际语言学、社会语言学, 等等。对于这一 点, 许国璋先生认为: “应用语言学是语言理论和语言教学的一道桥梁。” ${ }^{[8]}$ 事实确乎如此, 学者们已经将语言学相关理论方法及其研究成果应用于我国的语文教学实践中, 使之兴起了 一个新的学科—— “汉语应用语言学”。“汉语应用语言学” 的建立, 不仅对应用语言学乃 至普通语言学的学科建设具有重要的理论意义, 而且对于推动我国的语文教育教学改革, 大 面积提高语文教学效益, 增进中小学生语文能力发展, 提高全民族科学文化素质, 也具有非 常重要的现实价值。诚然, 语文教学不宜、也不可能将语言学理论照单全收, 而应从培养学 生交际与思维能力的实际需要出发, 择取其适用部分, 吸收其实用理论来构建语文教学科学 化的言语知识体系。

\section{2. 国家教育政策的大力扶持为其对接提供了根本保证}

国家教育部 2011 年制订颁布的《义务教育语文课程标准》指出：“语文课程是一门学习 语言文字运用的综合性、实践性课程。” ${ }^{[1]}$ 初中学生阅读应达到如下目标之一：了解基本的 语法知识, 以帮助理解课文中的语言难点; 了解常用的修辞方法, 体会它们在课文中的表达 作用。课标还在附录中专列 “语法修辞知识要点” , 以确定教学内容和范围。2013 年, 教育 部颁布的《普通高中语文课程标准 (实验) 》也明确指出: “高中语文课程应进一步提高学 生的语文素养, 使学生具有较强的语文应用能力……为终身学习和有个性的发展奠定基础。” ${ }^{[9]}$ 根据以上表述, 我们可以得出这样一个结论: 语文课标的一个重要理念就是重视语言基础 知识的积累, 这是全面提高学生语文素养的重要途径。

另外, 据我们调查, 近年来, 尽管语文高考题型不断发生变化, 但同语言学密切相关的 语言基础知识不可或缺, 一直是其中一个重要的组成部分。不仅如此, 而且其题型内容也越 来越灵活, 越来越注重考察学生的语言表达艺术和语言运用能力, 而这显然离不开教师语言 
学理论知识的引导。

\section{3. 高校合格人才的重点培养为其对接提供了现实基础}

众所周知, 现在中学教师很大部分来自高师院校, 高师院校开设的课程大多是为基础教 育服务的。而语言学不仅与语文教学密切相关, 还对语文教学有着重要的指导作用。因此, 高师院校相关的语言学课程, 是为提高语文教师语言理论素养而开设的。据调查, 江西大多 数高校诸如江西师范大学、赣南师范大学、上饶师范学院、江西科技师范大学、南昌师范学 院、井冈山大学、南昌大学科技学院、新余学院、宜春学院、九江学院等都开设了语言学理 论课。下面是这十所院校师范类语文专业课程设置的调查数据。

\section{江西十所院校师范类语文教育专业语言类课程设置调查数据}

\begin{tabular}{|c|c|c|c|c|c|c|}
\hline 现代汉语 & 古代汉语 & 普通话 & $\begin{array}{c}\text { 语言学概 } \\
\text { 论 }\end{array}$ & 训诂学 & 文字学 & 词汇学 \\
\hline 10 & 10 & 10 & 8 & 4 & 3 & 2 \\
\hline
\end{tabular}

从上表看出，高校师范类语文教育专业开设的语言相关课程虽然参差不齐，但都是基础 语文教学所必需的, 这就为语文教学输送了大量的合格人才, 它为语言学与语文教学实现有 效对接提供了良好的现实基础。

\section{4. 结语}

总而言之, 语言学与语文教学关系非常密切。实质上, 语文教学中的内容诸如语音、词 汇、语法、语义、文字、修辞、文体、写作、语用等方面, 均属语言学理论知识范畴之内, 是其不可分割的重要组成部分。早在 20 世纪 90 年代, 张志公先生就认为 “在语言学和语文 教学之间构建桥梁性学科是一个很值得进一步探索的课题” , 甚至还有一个要 “在语言学基 础知识、基础理论和语文教学之间建立一种桥梁性学科的构想”。 ${ }^{[10]}$ 由此看来, 将语言学理 论引入语文教学中实现有效对接, 并非是主观臆断的空中楼阁, 而是有其坚实的现实基础, 现在不仅显示出其必要性和可能性, 而且还是一件十分迫切的事情。如果真正实现了二者完 美而有效地对接, 它必将促使我国语文教育教学的大改革, 解决制约语文学科 “发展的薄弱 环节和突出瓶颈问题” ; ${ }^{[11]}$ 它也必将推动我国语文教育教学的大发展, 提高语文教学的社会 效益，增强中小学生的语文能力，从而全面提升整个民族的科学文化素质。

\section{致谢}

本文为中国江西省高等学校教学改革科研课题《高师语言学理论与基础语文教学实践问 题研究——关于如何加强高师语言学理论与基础语文教学实践对接问题的思考》（批准号 JXJG-13-2-4）的阶段性成果之一。

\section{References}

[1]People's Republic of China, Department of Education, Chinese Curriculum Standard of Compulsory Education (2011 edition), Beijing Normal University Press, pp.1-2, 2012.

[2]Xu Tongqiang, Ye Feisheng. The Outline of Linguistics, Peking University Press, pp. 5-6, page 2, 2010.

[3]Zhang Zhigong. On the Traditional Chinese EducationTeaching Theory and Materials, Shanghai Education Press, page 3, page 174, 1992.

[4]He Zhaoxiong. Journal of Pragmatics, Shanghai Foreign Language Education Press, page 12, 1989.

[5][America] Grice, Usage of Words, Foreign Language Teaching and Research Press, pp. 32-33, 2012. 
[6]Wang Yuanhua, The Chinese Teaching is Essentially Pragmatic Teaching, copy data from Renmin University of China (Chinese Teaching and Learning in Middle School), page 8, 2008 (11). [7]Wang Yuanhua, On the Chinese Teaching in the Perspective of Pragmatics, Beijing Normal University Press, page 25, 2012.

[8]Gui Shichun, The Introduction of English teaching in China and Self-study of Chinese Application Language, Shandong Education Press, page 2, 1988.

[9]People's Republic of China, Department of Education, Language Curriculum Standards of Ordinary High School (experimental edition), People's Education Press, page 2, 2013.

[10]Zhang, Zhigong. Zhang Zhigong's Study on Chinese - Public Opinion set (Wang Benhua edited), Chinese Press, page 25, 1998.

[11]Zhu Hong. Deepen the Reform of Undergraduate Education, Journal of Jiangxi Normal University (PHILOSOPHY AND SOCIAL SCIENCES EDITION), Vol.48, page 6, 2015 (04). 